\title{
A Case of Multifocal Musculoskeletal Tuberculosis in Pregnant Woman
}

\author{
Abeer Albaadania, c, Abdulaziz Al Anazi ${ }^{\mathrm{b}}$
}

\begin{abstract}
Multidrug-resistant tuberculosis (MDR-TB) is a major health problem affecting childbearing age women. Here we present a very rare entity of TB in a pregnant woman involving multifocal skeletal system treated with second-line anti-TB according to culture susceptibility pattern. On completion of therapy, patient showed good clinical response.
\end{abstract}

Keywords: Tuberculosis; Pregnancy; Resistance

\section{Introduction}

Multidrug-resistant tuberculosis (MDR-TB) has emerged from different parts of the world secondary to variable situations. In 2013, WHO estimated about $3.7 \%$ of new TB patients in the world have MDR-TB. Incidence is higher in those previously treated, about $20 \%$. The frequency of MDR-TB varies substantially between countries with most cases occurring in Asia (58\%) and Africa (27\%) [1]. In 2013, Al-Hajoj et al recorded the results of the first nationwide drug resistance survey conducted in Saudi Arabia that showed a low level (4\%) of MDR-TB, whereas the rate of any drug resistance was found to be $23.6 \%$ [2]. A treatment history of active TB, being foreign-born, having pulmonary $\mathrm{TB}$, and living in the Western part of the country were the strongest independent predictors of MDR-TB from the national survey data collected between August 2009 and July 2010 [3]. Gulf Cooperation Council (GCC) member states risk of MDR-TB was found to be almost equivalent to other developing or developed countries and iso-

Manuscript accepted for publication July 07, 2016

aDivision of Infectious Disease, Department of Medicine, Prince Sultan Military Medical City, Riyadh, Saudi Arabia

bepartment of Medicine, Prince Sultan Military Medical City, Riyadh, Saudi Arabia

${ }^{\mathrm{c} C}$ Corresponding Author: Abeer Albaadani, Division of Infectious Disease, Department of Medicine, Riyadh Prince Sultan Military Medical City, Riyadh, Saudi Arabia. Email: albaadani.id@gmail.com

doi: http://dx.doi.org/10.14740/jmc2585w niazide resistance was found to be the strongest predictor for the identification of MDR-TB in all GCC countries [4].

\section{Case Report}

In October 2010, the patient was a 35-year-old pregnant woman at 24 weeks gestational age, mother of three children (child aged 2 years and twins aged 10 months). Her obstetric history revealed nine previous abortions labeled as probable anti-phospholipid syndrome and commenced on low molecular weight (LMW) heparin. She lives at home with her husband, children and two maids and works as admin staff at a Riyadh Hospital, Saudi Arabia. She presented to orthopedics clinic with left ankle progressive pain, remembering twisting her ankle had associated with the onset of that. She reported possible occasional fever, no cough, no weight loss, no night sweats, and no history of similar illness before.

On examination, she had no fever, swollen left ankle, hyperemic skin, cold to touch, and mildly tender, fluctuant, probable abscess (cold) (Fig. 1).

Her laboratory results showed white blood cell count (WBC) of $8.3 \times 10^{9} / \mathrm{L}$, neutrophils of $6.4 \times 10^{9} / \mathrm{L}$, and CRP of $166 \mathrm{mg} / \mathrm{L}$. Plain foot X-ray and ultrasound revealed noncommunicating hypoechoic soft tissue masses.

The maximum lesion size was about $3 \times 2.7 \times 2 \mathrm{~cm}$ located at the anterior medial aspect of the foot dorsum. Color Doppler study showed significant mixed internal flow in the lesions indicating that these were solid masses rather than collection (Figs. 2 and 3).

MRI showed large macrolobulated soft tissue mass suggestive of soft tissue sarcoma (Fig. 4). Ultrasound-guided

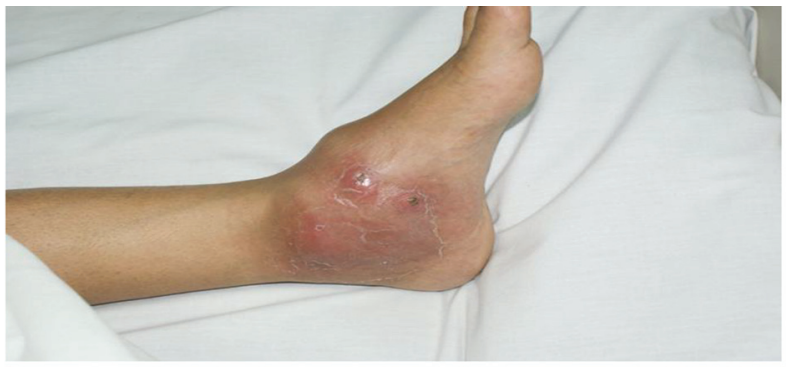

Figure 1. Physical examination of the left ankle joint. 


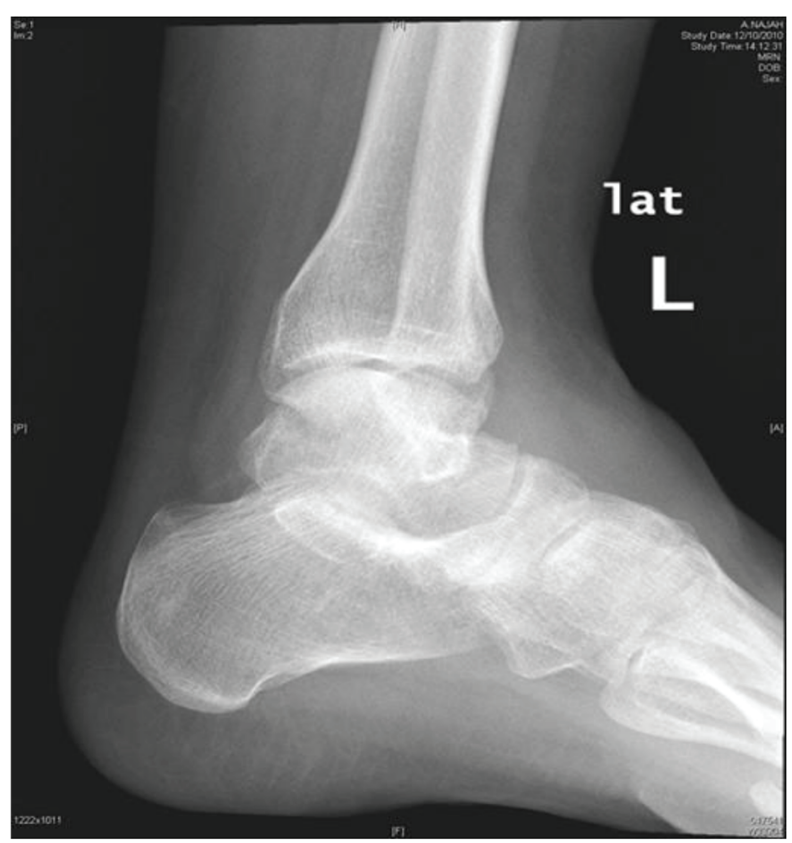

Figure 2. Plain X-ray of left ankle.

biopsy histopathology showed necrotizing granulomatous inflammation consistent with TB (Fig. 5).

Based on clinical, radiological and histological background, she was commenced on standard quadruple anti-TB therapy: rifampicin, isoniazid, pyrazinamide and ethambutol, waiting for the final microbiological result. She was taken back to theater by orthopedics for further debridement; surgeons described seeing "caseating material". After a period of recovery including pain control and dressings, she was discharged with clinic follow-up.

After 1 month period, she came back to the clinic and reported fever, anorexia and continuing pain and swelling in spite of perfect adherence and good tolerance to anti-TB. Culture was still in progress, and she was given another appointment within 2 weeks when she complained of lump in the back, back and shoulder pain and continuing complaints of anorexia, documented weight loss yet no neurological deficit. She was a reliable patient, with no concerns over compliance

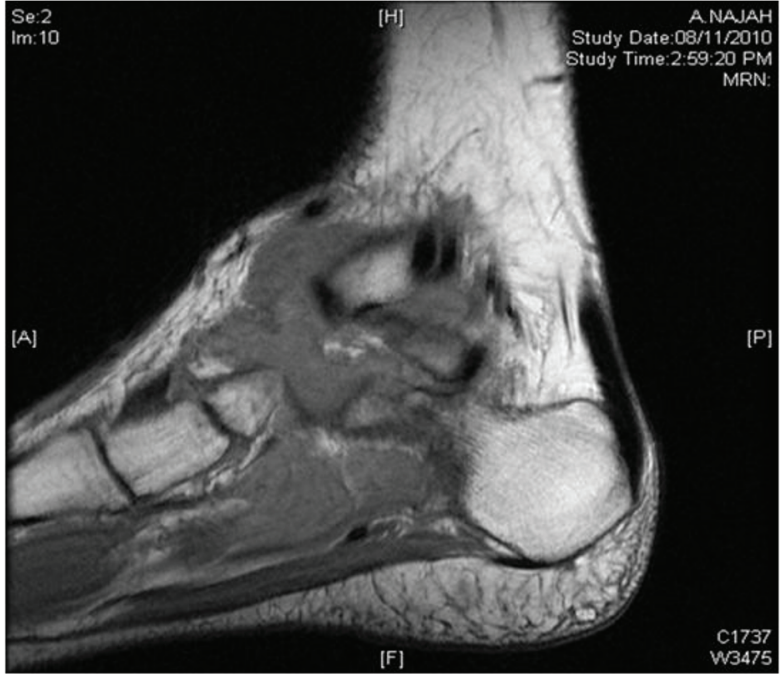

Figure 4. MRI of left ankle shows macrolobulated mass.

(Fig. 6).

By that time, culture results were available which showed Mycobacterium tuberculosis resistant to all first-line anti-TB including streptomycin. Then she was admitted to the isolation ward, induced sputa for Ziehl-Nelson stain $\times 3$ was ordered which was negative, and all anti-TB had been discontinued with urgent MRI spine which showed paraspinal large anterior soft tissue collection extending from the level of T8 to L1 with evidence of osteomyelitis as well as multiple enlarged lymph nodes noted within the superior mediastinum (Fig. 7).

Further anti-TB susceptibility results were obtained from abroad center Table 1 .

Multi-disciplinary plan was arranged between adult infectious diseases, obstetrician, pediatric infectious diseases, neonatologist medical microbiologist and clinical pharmacists. Patient underwent elective cesarean section at 28/40 weeks, and gave well newborn who stayed in neonatal intensive care unit (NICU). After the fetus went safely out, she was put on pyrazinamide, amikacin, moxifloxacin, cycloserine and linezolid. Over a period of 3 months on the stated regimen, nausea/vomiting, poor dietary intake, oral thrush, stomatitis, anemia, anxiety and depression were recorded. She was man-

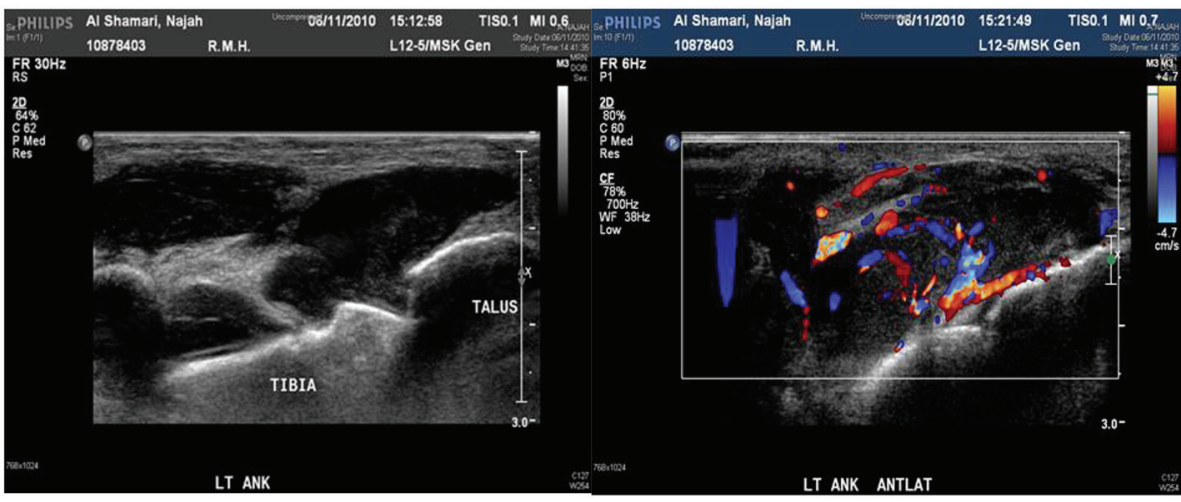

Figure 3. Ultrasound and Doppler ultrasound of left ankle show about $3 \times 2.7 \times 2 \mathrm{~cm}$ collection. 


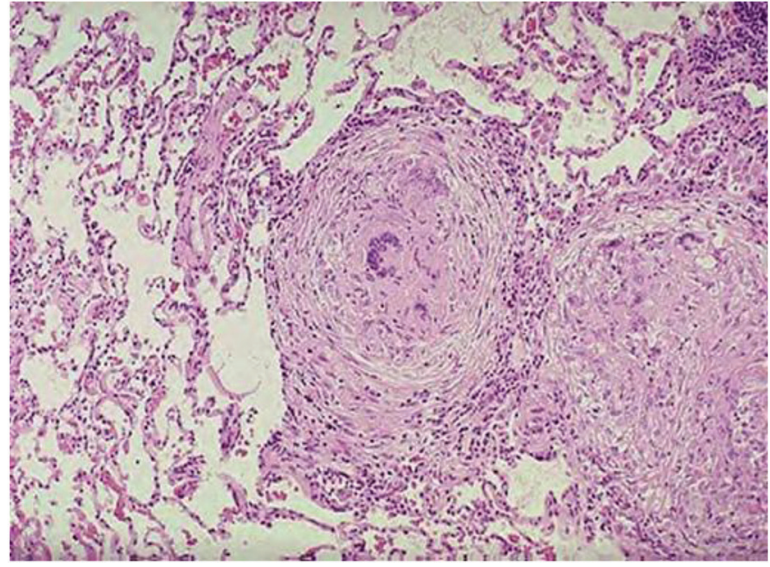

Figure 5. Histopathology shows necrotizing granulomatous.

aged to be discharged on five anti-TB agents pyrazinamide, streptomycin, moxifloxacin, cycloserine, linezolid with supportive treatment (anti-emetics, pain killers, pregabalin and dietary supplements). After 1 month, she was seen in the clinic still having ongoing poor intake, failure to gain weight and progressive peripheral neuropathy. Remarkably, there was no hearing impairment (formal audiology) or nephrotoxicity. Ankle and paraspinal collection had resolved. But she developed linezolid-induced pancytopenia ( $\mathrm{Hb} 10.0 \mathrm{~g} / \mathrm{L}$, WCC 3.0 $\times 10^{9} / \mathrm{L}$ and platelets $\left.11 \times 10^{9} / \mathrm{L}\right)$. Linezolid discontinued and was put in nasogastric tube feeding anti-emetic and assessed by the psychiatry who labeled her having "adjustment disorder" for which alprozolam was started. When the blood count had recovered and improved tolerance, linezolid re-instated and discharged home. Streptomycin has been discontinued after 5 months and continued on pyrazinamide, moxifloxacin, and cycloserine. Later on, given the neuropsychiatric side effect of cycloserin, it was switched to para-aminosalicylates (PAS). All anti-TB had been stopped after 21 months of therapy. She was well with no evidence of active TB and back to the work fully active.



Figure 6. Back lump after 2 months of first-line anti-TB.

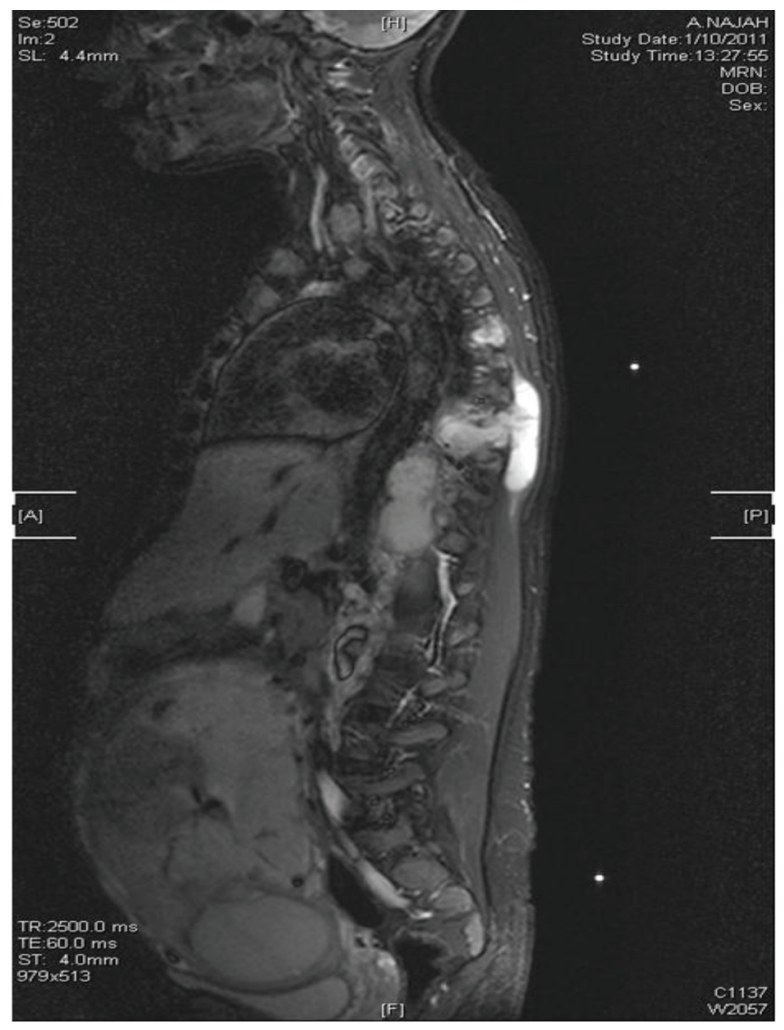

Figure 7. MRI of spine T8-L1 osteomyelitis and paraspinal collection.

\section{Discussion and Literature Review}

Globally, TB is the third leading cause of morbidity and premature mortality in childbearing age women $[5,6]$. The risk of $\mathrm{TB}$ is increasing in pregnant woman from $0.5 \%$ to $11 \%$ if

Table 1. Anti-TB Susceptibility Result From Two Centers

\begin{tabular}{llll}
\hline Available & Agent & Riyadh & London \\
\hline Yes & Rifampicin & $\mathrm{R}$ & $\mathrm{R}$ \\
Yes & Isoniazide & $\mathrm{R}$ & $\mathrm{R}$ \\
Yes & Pyrazinamide & $\mathrm{R}$ & $\mathrm{S}$ \\
Yes & Ethambutol & $\mathrm{R}$ & $\mathrm{R}$ \\
Yes & Streptomycin & $\mathrm{S}$ & $\mathrm{S}$ \\
Yes & Amikacin & $\mathrm{S}$ & $\mathrm{S}$ \\
Yes & Ciprofloxacin & $\mathrm{S}$ & - \\
Yes & Moxifloxacin & - & $\mathrm{S}$ \\
No & Capreomycin & $\mathrm{S}$ & $\mathrm{S}$ \\
Yes & Ethionamide & $\mathrm{S}$ & $\mathrm{R}$ \\
No & Kanamycin & $\mathrm{S}$ & $\mathrm{S}$ \\
Yes & Linozolid & - & $\mathrm{S}$ \\
Yes & Cycloserin & - & - \\
No & PAS & - & - \\
\hline
\end{tabular}

PAS: para-aminosalicylate. 
co-infected with HIV in high burden area [7]. Owing to the hormonal changes during pregnancy secondary to cortisol upregulations, the progression from latent to active TB is higher in pregnant female than in non-pregnant [8]. Most of the reported cases of MDR-TB in pregnant were based on retrospective case series or case reports from different parts from the world (India and Lima), mostly pulmonary TB [9-11]. Rezai et al had reported extrapulmonary with intraocular dissemination of MDR-TB of pregnant woman at 28 weeks gestational age [12]. The prevalence of extrapulmonary TB (EXPTB) in the Eastern Mediterranean region (which includes Saudi Arabia), according to the $\mathrm{WHO}$, is $22 \%$. It is noteworthy Saudi Arabia exceeded the average (28\% in 2012). However, there are no documented data in pregnancy [13]. EXPTB is usually more common among the immune compromised patients, and pregnancy is one factor which can relatively affect the T-cell immunity that increases the risk of TB. Nevertheless, the reported EXPTB MDR-TB in pregnancy is very uncommon.

Our patient presented with ankle joint swelling with denial of any chronic constitutional symptoms that did not have high clinical suspicion and radiological evidence of TB until the histopathology result became available. The lack of improvement on standard anit-TB drugs with progression to the spine had put the MDR-TB of the ankle, ribs, scapula and the spinal bone high possibility which was then confirmed by the microbiological susceptibility test. Skeletal TB constitutes 10 $20 \%$ of all the EXPTB. It is clinical condition that is easily diagnosed and managed with an excellent outcome [14]. Musculoskeletal TB had been reported from British review study over 6 years, revealing 61 cases $(8.4 \%)$ out of 729 cases of TB notifications. Of the patients, $74 \%$ were immigrants from the Indian subcontinent; nearly $50 \%$ had spinal involvement [15]. Another study from Nigeria reported the prevalence of musculoskeletal TB about $0-15 \%$ of all TB notification in nonindustrialized world [16]. Interestingly, our patient had multifocal bone involvement secondary to TB which considered a very rare variant of skeletal $\mathrm{TB}$, it constitutes $<5 \%$ of all skeletal TB cases even in high TB endemic area $[17,18]$. To our knowledge, our case is the first case report in the literature describing multifocal musculoskeletal involvement MDR-TB in pregnant woman. Multidisciplinary teamwork should be applied to address the patient concerns regarding the prognosis and the outcome for her and her fetus. There is no evidence that $\mathrm{TB}$ is more common or more serious in pregnant women than non-pregnant women. Moreover, pregnancy does not increase the risk of relapse or worsen TB in patients who are treated adequately. However, TB is considered a serious and potentially deadly risk to the fetus and newborn baby if it is not detected early and treated properly in the mother [19, 20]. Meta-analysis has shown that the proportion of patients with MDR-TB treated successfully improved when treatment duration was at least 18 months, and if patients received directly observed therapy throughout treatment at the same time, data showed that individualized treatment regimens had higher treatment success than standardized regimens [21]. We faced difficulties in the management of this patient due to several aspects: being pregnant, having multifocal musculoskeletal TB involvement, and different anti-TB susceptibility testing in several occasions. Subjecting the patient to more toxic, pos- sible teratogenic and less effective drugs was a major decision to outweigh the risk of the adverse effects of maternal $\mathrm{TB}$ on fetus and the risk of the adverse effects of maternal TB treatment on fetus. Most of the literature addressing the treatment of MDR-TB in pregnancy is based on case reports or case series. Largest is a cohort study from Lima, Puru, that highlighted the treatment outcomes of 38 cases. After completion of MDR-TB treatment, more than $50 \%$ of women have cured and $5 \%$ had experienced treatment failure [9]. The safety of the seconded-line anti-TB varies in pregnancy between class $\mathrm{C}$ as with flouroquinolones, ethionamide and cycloserin to class D as with aminoglycosides. It is worth to mention we managed to induce labor at 28 weeks to our patient, then we started the best available treatment with combination of pyrazinamide, streptomycin, moxifloxacin, cycloserine and linezolid as she continued the injectable regimen for 5 months, and then she completed the course with pyrazinamide, moxifloxacin and cycloserine. However, given the poor neuropsychiatry side effect of cycloserin, it had to be changed to PAS. The duration was 21 months which is comparable to the duration mentioned in the literature of 18 - 24 months with favorable outcome to the mother and her new born who was kept in the nursery incubator with healthy condition with no evidence of TB in placenta or gastric aspirate.

\section{Acknowledgments}

I express my deep feeling of gratitude and I am very much thankful to Dr Ali AL Omrani for his supervision and guidance in the this patient's management.

\section{References}

1. www.who.int/tb/challenges/mdr24/4/2016

2. Varghese B, Supply P, Shoukri M, Allix-Beguec C, Memish Z, Abuljadayel N, Al-Hakeem R, et al. Tuberculosis transmission among immigrants and autochthonous populations of the eastern province of Saudi Arabia. PLoS One. 2013;8(10):e77635.

3. Al-Hajoj S, Varghese B, Shoukri MM, Al-Omari R, AlHerbwai M, Alrabiah F, Alrajhi AA, et al. Epidemiology of antituberculosis drug resistance in Saudi Arabia: findings of the first national survey. Antimicrob Agents Chemother. 2013;57(5):2161-2166.

4. Areeshi MY, Bisht SC, Mandal RK, Haque S. Prevalence of drug resistance in clinical isolates of tuberculosis from GCC: a literature review from January 2002 to March 2013. J Infect Dev Ctries. 2014;8(9):1137-1147.

5. Clark PC, Yencha MW, Hart AK. Management of isolated extrapulmonary tuberculosis in a pregnant patient. Ann Otol Rhinol Laryngol. 2004;113(8):648-651.

6. Zenner D, Kruijshaar ME, Andrews N, Abubakar I. Risk of tuberculosis in pregnancy: a national, primary carebased cohort and self-controlled case series study. Am J Respir Crit Care Med. 2012;185(7):779-784.

7. Mathad JS, Gupta A. Tuberculosis in pregnant and postpartum women: epidemiology, management, and research 
gaps. Clin Infect Dis. 2012;55(11):1532-1549.

8. Allolio B, Hoffmann J, Linton EA, Winkelmann W, Kusche M, Schulte HM. Diurnal salivary cortisol patterns during pregnancy and after delivery: relationship to plasma corticotrophin-releasing-hormone. Clin Endocrinol (Oxf). 1990;33(2):279-289.

9. Palacios E, Dallman R, Munoz M, Hurtado R, Chalco K, Guerra D, Mestanza L, et al. Drug-resistant tuberculosis and pregnancy: treatment outcomes of 38 cases in Lima, Peru. Clin Infect Dis. 2009;48(10):1413-1419.

10. Rohilla M, Joshi B, Jain V, Kalra J, Prasad GR. Multidrug-Resistant Tuberculosis during Pregnancy: Two Case Reports and Review of the Literature. Case Rep Obstet Gynecol. 2016;2016:1536281.

11. Shin S, Guerra D, Rich M, Seung KJ, Mukherjee J, Joseph K, Hurtado R, et al. Treatment of multidrug-resistant tuberculosis during pregnancy: a report of 7 cases. Clin Infect Dis. 2003;36(8):996-1003.

12. Rezai S, LoBue S, Adams D, Oladipo Y, Posso R, Mapp T, Santiago C, et al. Untreated Active Tuberculosis in Pregnancy with Intraocular Dissemination: A Case Report and Review of the Literature. Case Rep Pulmonol. 2015;2015:370462.

13. World Health Organization. Global Tuberculosis Report 2013. Geneva: WHO. 2013.

14. Moon MS, Moon YW, Moon JL, Kim SS, Sun DH. Con- servative treatment of tuberculosis of the lumbar and lumbosacral spine. Clin Orthop Relat Res. 2002;398:4049.

15. Talbot JC, Bismil Q, Saralaya D, Newton DA, Frizzel RM, Shaw DL. Musculoskeletal tuberculosis in Bradford - a 6-year review. Ann R Coll Surg Engl. 2007;89(4):405409.

16. Iyidobi EC, Nwadinigwe CU, Ekwunife RT. Management of Musculoskeletal Tuberculosis in Enugu, Nigeria. Trop Med Surg. 2013;1:156.

17. Yilmaz MH, Kantarci F, Mihmanli I, Kanberoglu K. Multifocal skeletal tuberculosis. South Med J. 2004;97(8):785787.

18. Marudanayagam A, Gnanadoss JJ. Multifocal skeletal tuberculosis: a report of three cases. Iowa Orthop J. 2006;26:151-153.

19. Davidson PT. Managing tuberculosis during pregnancy. Lancet. 1995;346(8969):199-200.

20. Carter EJ, Mates S. Tuberculosis during pregnancy. The Rhode Island experience, 1987 to 1991. Chest. 1994;106(5):1466-1470.

21. Orenstein EW, Basu S, Shah NS, Andrews JR, Friedland GH, Moll AP, Gandhi NR, et al. Treatment outcomes among patients with multidrug-resistant tuberculosis: systematic review and meta-analysis. Lancet Infect Dis. 2009;9(3):153-161. 